\title{
Physical Activity and Respiratory Muscle Strength in Patients with Sarcoidosis: An Observational
}

\section{Study}

\author{
Camelia Pescaru' \\ Mirela Frandes (D) ${ }^{2}$ \\ Monica Marc id \\ Daniel Traila' \\ Andrei Pescaru' \\ Cristian Oancea' \\ 'Department of Pulmonology, "Victor \\ Babes" University of Medicine and Pharmacy, \\ Timisoara, Romania; ' 2 Department of \\ Functional Sciences - Medical Informatics \\ and Biostatistics, "Victor Babes" University \\ of Medicine and Pharmacy, Timisoara, \\ Romania
}

Correspondence: Mirela Frandes

Department of Functional Sciences - Medical Informatics and Biostatistics, "Victor Babes" University of Medicine and Pharmacy, 2

Eftimie Murgu, Timisoara, 30004I, Romania Tel +40731 I I 7020

Email mirela.frandes@umft.ro
Background: The literature results related to the physical assessment in patients with sarcoidosis are contradictory and limited. The objective of this study was to compare the physical activity and respiratory muscle strength of patients at different stages of sarcoidosis. Methods: Since 2003, we monitored 386 patients with ILDs, from which 107 patients were with sarcoidosis. Of the 107 patients with sarcoidosis, 57 were included in this study. A matched control group of 50 healthy patients were also included in the study. Kings Sarcoidosis Questionnaire (KSQ), maximal inspiratory/expiratory pressure and 6-minute walking test were recorded.

Results: We observed significant differences between the exercise capacity of the four sarcoidosis stages, $561.00(531.00-586.00)$ vs $557.00(510.00-599.00)$ vs 469.00 (430.00$515.00)$ vs 457.00 (397.00-496.00), independent-samples Kruskal-Wallis $H$-test $H(4107)=$ $34.757, p<0.001$. Also, we observed significant differences between the exercise capacity of patients with sarcoidosis and healthy controls, 496.00 (437.00-550.00) vs 561.00 (531.00586.00), Mann-Whitney $U$-test, $p<0.001$. In addition, we observed that patients with sarcoidosis had significantly lower MIP and MEP than healthy controls, 84.00 (79.00$91.00)$ vs $90.00(83.00-97.00) ; \mathrm{p}=0.001$, and $85.00(79.00-89.00)$ vs $89.00(85.00$ $92.00), \mathrm{p}<0.001$, respectively. There were significant associations between exercise capacity and KSQ scores, eg, general health $(r=0.206, p=0.027)$, lung health $(r=0.280, p=0.003)$. Conclusion: Patients with different stages of sarcoidosis showed reduced quality of life, exercise capacity and respiratory muscle strength both among themselves and compared to healthy controls.

Keywords: exercise capacity, sarcoidosis, muscle strength, Kings Sarcoidosis Questionnaire, 6-minute walking test

\section{Background}

Sarcoidosis is a systemic granulomatous disease of unknown aetiology characterized by the formation of epithelioid cell granuloma without caseation that mainly affects the lungs and lymphatic system. ${ }^{1}$

Frequently affected by the disease are the lungs, skin, eyes and musculoskeletal system but the lungs are affected in $90 \%$ of patients. Patients often have nonspecific symptoms, such as general weakness, arthralgia and reduced exercise capacity. ${ }^{2}$ Limited exercise tolerance could be explained by peripheral muscle weakness and exercise intolerance caused by fatigue and both can be caused by several factors, such as decreased lung function, negative vicious circle of physical deconditioning, and corticosteroid-induced myopathy. ${ }^{3}$ 
Studies have reported inspiratory muscle weakness in patients with sarcoidosis ranging from $16.7 \%$ up to $45 \%$ and expiratory muscle weakness has been found to be decreased up to $40 \% .^{3-6}$

Correlations have been observed between reduced respiratory muscle strength and exercise-induced dyspnea, especially in the advanced stages of the disease. ${ }^{4,5}$ Moreover, Kabitz et al demonstrated that inspiratory muscle strength is strongly predictive for dyspnea and functional exercise capacity. ${ }^{4}$

The 6-minute walk test (6MWT) is widely used to evaluate exercise capacity and previous studies have found that the distance walked in the test has been reduced in patients with sarcoidosis compared to healthy subjects. ${ }^{7,8}$

Some authors are reporting that impaired inspiratory muscle strength is an important factor in the reduced sixminute walking distance (6MWD). ${ }^{4}$ Two studies showed that 6 MWD was reduced $(<400 \mathrm{~m})$ in the majority of patients with sarcoidosis. This was caused by skeletal muscle weakness or reduced peripheral muscle strengths. ${ }^{7,8}$ While these authors found that the limiting factor was skeletal muscle weakness or reduced peripheral muscle strength, Wirnsberger et al found that the limiting factor was reduced respiratory strength and endurance time. ${ }^{6}$

However, studies on physical activity measured objectively in patients with sarcoidosis are quite limited, and the results are contradictory. One author found that physical activity and fatigue are reduced in patients with sarcoidosis compared to predicted values, ${ }^{9}$ while Saligan did not find significant differences between patients with sarcoidosis and control subjects. ${ }^{10}$

Given the fact that literature results are contradictory and physical assessment in patients with sarcoidosis remains limited, the objective of this study was to assess and compare the physical activity and respiratory muscle strength of patients with different stages of sarcoidosis.

\section{Materials and Methods Study Design and Patients}

This was a retrospective cohort study in which patients were included from a database for interstitial lung diseases of the hospital "Victor Babes" in Timisoara. Since 2003, 386 patients were included from which 107 patients are with sarcoidosis. The diagnosis for sarcoidosis was established according to the guidelines: after the clinical exam, chest radiography or computer tomography scan. ${ }^{11}$
Histopathologic examination was performed on patients with insufficient clinical-imagistic features for a confident diagnostic The division on stages has been made according to chest radiography and/or computer tomography. ${ }^{11}$

From the 107 patients with sarcoidosis, 57 were included in this study. A number of 50 patients were excluded due to the lack of several tests such as King 's Sarcoidosis Questionnaire (KSQ), Maximal Inspiratory/ Expiratory Pressure test (MIP/MEP) or 6MWT. All the tests were performed when the patient's diagnosis was confirmed and according to the American Thoracic Society (ATS) guideline. ${ }^{12,13}$ The control group was formed of healthy volunteers who agreed to participated in the study. The study was approved by the ethic committee of the hospital (nr.6241/24/08/2020).

\section{Assessing Lung Function}

To determine the lung function, patients performed spirometry and diffusing capacity of lung for carbon monoxide (DLCO) test using the COSMED Q-Box and Quark COSMED. ${ }^{12}$

\section{Assessing Functional Capacity}

To evaluate the functional capacity, patients performed the 6MWT which measures the distance an individual can walk in six minutes. It is a simple test that requires no special equipment or advanced training for the physicians and is considered to be the substitute of the gold standard cardiopulmonary exercise test (CPET). ${ }^{14}$

Respiratory muscle strength was determined with the COSMED Q-Box device by measuring the MIP and MEP. These measures are simple, non-invasive, and have established diagnostic and prognostic value. Maximal Inspiratory Pressure determines the strength mainly of the diaphragm and other inspiratory muscles, while Maximal Expiratory Pressure determines the strength of mainly the abdominal muscles and other expiratory muscles. ${ }^{15}$

\section{Assessing Dyspnea and Exertion}

The modified Borg scale is a 0 to 10 rated numerical score used to measure dyspnea and leg fatigue reported by the patient. ${ }^{16}$

\section{Assessing Patients' General Health}

The impact of sarcoidosis on various aspect of the patient's life was determined with the King`s Sarcoidosis 
Questionnaire, which is a validated questionnaire for patients with sarcoidosis. ${ }^{17}$ The questions are related to how sarcoidosis has affected the patients' health. The KSQ is a self-administered questionnaire composed of 29 questions in total, divided into 5 categories: General Health (10 items), Lung (6 items), Skin (3 items), Eye (7 items) and Medications (3 items). Each item is expressed by a Likert scale with 7-points. Results are given as a number between $1-100$ with higher numbers indicating better health. ${ }^{17}$

\section{Statistical Analysis}

Statistical data analysis was performed using the SPSS v.20 software (SPSS Inc., Chicago, IL, USA). The graphical representations were done using GraphPad Prism v. 9.1 software. The statistical significance was established considering the threshold $p$-value $<0.05$, and, for estimating intervals, a value of 0.95 was considered as the confidence level.

Continuous data with normal distribution were described as mean ( \pm standard deviation), while continuous data with other distributions were presented as median (interquartile range). Absolute frequency (percentage) was used to describe nominal data. The distributions of continuous data were checked for normality by applying the Kolmogorov-Smirnov's test, while the Levene's test was applied to verify the equality of variances.

The differences between groups of patients with sarcoidosis were tested for statistical significance using the Student's $t$-test. When data was not normally distributed, the difference between groups was tested for statistical significance using the Wilcoxon signed-rank test for related-samples or the Mann-Whitney $U$-test for independent samples. The statistical significance of the difference between percentages was assessed applying the Pearson chi-square or Fisher's exact test. When comparing more than two groups of patients with sarcoidosis, we applied one-way ANOVA for continuous data with Gaussian distribution or Kruskal-Wallis $H$-test for continuous data with non-Gaussian distribution. The Spearman correlation coefficient was computed to assess the association between non-Gaussian distributions measured at the same point in time.

\section{Results}

\section{Patients' Characteristics}

The sample included 57 patients with sarcoidosis, aged between 21 and 71 years, mean $43.56( \pm 12.3)$ years, 50.9\% men and $49.1 \%$ women. A percent of $33.3 \%$ of the patients with sarcoidosis were in the third stage (pulmonary). We also considered a matched control group of 50 healthy individuals, aged between 21 and 71 years, mean $43.56( \pm 12.3)$ years, 50.9\% men and 49.1\% women (Table 1).

The pulmonary function of patients was tested by measuring the spirometry parameters, the lung volumes, the maximal respiratory pressures, and the diffusing capacity. We observed that the results of spirometry and lung tests of patients with sarcoidosis were not significantly different than the results of the healthy controls. The maximal respiratory pressures were significantly lower in patients with sarcoidosis than in healthy controls (Table 2).

We observed that both MIP and MEP were significantly lower in patients with sarcoidosis than in healthy controls (Figure 1).

There were significant differences between the exercise capacity of the four sarcoidosis stages, 561.00 (531.00$586.00)$ vs $557.00(510.00-599.00)$ vs 469.00 (430.00515.00) vs 457.00 (397.00-496.00), independent-samples Kruskal-Wallis $H$-test $H(4107)=34.757, p<0.001$. At the same time, we observed significant differences between the exercise capacity of patients with sarcoidosis and healthy controls, 496.00 (437.00-550.00) vs 561.00 (531.00-586.00), Mann-Whitney $U$-test, $p<0.001$ (Table 2, Figure 2).

We also measured the Borg dyspnea score, and the Borg fatigue score before and after the 6MWD test. In the case of patients with sarcoidosis, we observed that the Borg dyspnea score and the Borg fatigue score after 6MWD were significantly higher than before 6MWD (related-samples Wilcoxon signed-rank test, $z=6.540$,

Table I Characteristics of Patients with Sarcoidosis and Healthy Controls

\begin{tabular}{|c|c|c|c|}
\hline Parameters & $\begin{array}{l}\text { Patients } \\
(n=57)\end{array}$ & $\begin{array}{l}\text { Controls } \\
(n=50)\end{array}$ & $p$-value \\
\hline Age & $43.56( \pm 12.3)$ & $42.08( \pm 11.28)$ & 0.517 \\
\hline Gender [male] & $29(50.9 \%)$ & $23(46 \%)$ & 0.615 \\
\hline Height (cm) & $170.28( \pm 9.11)$ & $169.04( \pm 8.4 \mathrm{I})$ & 0.570 \\
\hline Weight (kg) & $73.09( \pm 14.77)$ & $72.48( \pm 14.84)$ & 0.967 \\
\hline BMI $\left(\mathrm{kg} / \mathrm{m}^{2}\right)$ & $25.27( \pm 4.99)$ & $25.52( \pm 5.54)$ & 0.800 \\
\hline \multicolumn{4}{|l|}{ Stage } \\
\hline Stage I - lymphadenopathy & $19.3 \%$ & NA & NA \\
\hline $\begin{array}{l}\text { Stage II - lymphadenopathy } \\
\text { and pulmonary infiltrates }\end{array}$ & $22.8 \%$ & NA & NA \\
\hline Stage III - pulmonary infiltrates & $33.3 \%$ & NA & NA \\
\hline Stage IV - pulmonary fibrosis & $24.6 \%$ & NA & NA \\
\hline
\end{tabular}

Notes: Data are presented as mean ( \pm std. dev.) or absolute frequency (percentage)

Abbreviation: NA, not applicable. 
Table 2 Results of Pulmonary Function Testing of Patients with Sarcoidosis and Healthy Controls

\begin{tabular}{|c|c|c|c|}
\hline Tests & Patients $(n=57)$ & Controls $(n=50)$ & $p$-value \\
\hline \multicolumn{4}{|l|}{ Spirometry } \\
\hline FVC (L) & $3.57(3.10-4.19)$ & $4.03(3.57-4.62)$ & 0.002 \\
\hline FVC (\%) & $84.00(78.00-90.00)$ & $92.50(89.00-103.00)$ & $<0.001$ \\
\hline $\mathrm{FEV}_{\mathrm{I}}(\mathrm{L})$ & $2.85(2.21-3.26)$ & $3.21(2.99-3.69)$ & $<0.001$ \\
\hline $\mathrm{FEV}_{\mathrm{I}}(\%)$ & $80.00(69.00-86.00)$ & $94.00(85.00-100.00)$ & $<0.001$ \\
\hline $\mathrm{FEV}_{1} / \mathrm{FVC}(\mathrm{L})$ & $78.00(74.00-82.00)$ & $80.00(77.00-87.00)$ & 0.044 \\
\hline \multicolumn{4}{|l|}{ Lung volumes } \\
\hline $\mathrm{TCL}(\mathrm{L})$ & $83.00(71.00-91.00)$ & $89.00(85.00-97.00)$ & 0.001 \\
\hline $\mathrm{RV}(\mathrm{L})$ & $83.00(74.00-92.00)$ & $90.50(83.00-97.00)$ & 0.001 \\
\hline \multicolumn{4}{|c|}{ Maximal respiratory pressures } \\
\hline MIP (cmH2O) & 87.51 (78.57-100.52) & $90.99(78.14-111.96)$ & 0.002 \\
\hline MIP (\%) & $84.00(79.00-91.00)$ & $90.00(83.00-97.00)$ & 0.001 \\
\hline MEP $(\mathrm{cmH} 2 \mathrm{O})$ & 95.46 (76.10-109.73) & $92.12(78.85-114.10)$ & 0.009 \\
\hline MEP (\%) & $85.00(79.00-89.00)$ & $89.00(85.00-92.00)$ & $<0.001$ \\
\hline \multicolumn{4}{|c|}{ Diffusing capacity } \\
\hline DLCO (\%) & $20.43(18.45-24.82)$ & $26.13(21.31-36.24)$ & $<0.001$ \\
\hline $\mathrm{DLCO}_{\text {corr }}(\%)$ & $84.00(70.00-91.00)$ & $92.50(89.00-94.00)$ & $<0.001$ \\
\hline \multicolumn{4}{|l|}{ Physical activity } \\
\hline 6MWD (m) & $496.00(437.00-550.00)$ & $561.00(531.00-586.00)$ & $<0.001$ \\
\hline $6 M W D_{\text {pred }}(\%)$ & $93.00(86.00-96.00)$ & $97.50(93.00-100.00)$ & $<0.001$ \\
\hline
\end{tabular}

Notes: Data are presented as median (inter-quartile range).

Abbreviations: FEVI, forced expiratory volume in the first second; FVC, forced vital capacity; RV, residual volume; TLC, total lung capacity; MIP, maximal inspiratory pressure; MEP, maximal expiratory pressure; DLCO, diffusing capacity of lung for carbon monoxide; 6MWD, six-minute walking distance.

$p<0.001$, and $z=5.713, p<0.001$, respectively). In the case of healthy controls, we also observed that the Borg dyspnea score and the Borg fatigue score before 6MWD were significantly lower than after 6MWD (relatedsamples Wilcoxon signed-rank test, $z=5.990, p<0.001$, and $z=4.557, p<0.001$, respectively).

We investigated the health status of patients with sarcoidosis by analysing the KSQ scores. We observed that the health status of the patients with sarcoidosis was moderate to severely impaired (Table 3 ).

\section{Lung Function}

There was a significant association between exercise capacity measured by 6MWD and lung function measures in patients with sarcoidosis (FEV1: $r=0.432 * *, \mathrm{p}<0.001$; FVC: $\left.r=0.445^{* *}, p<0.001\right)$. There was no significant association between exercise capacity and lung volumes in patients with sarcoidosis (TCL: $r=0.175, p=0.056$; RV: $r=0.081$, $p=0.374)$. However, we observed that exercise capacity significantly correlated with diffusing capacity (DLCO: $\left.r=0.350^{* *}, p<0.001 ; \mathrm{V}_{\mathrm{A}}: r=0.276^{* *}, p=0.03\right)$. Also, exercise capacity significantly correlated with respiratory muscle strength in patients with sarcoidosis (MIP: $r=0.325^{*}$, $p=0.014$; MEP: $r=0.229^{*}, p=0.039$ ) (Figure 3).

\section{Health Status}

There were significant associations between exercise capacity and KSQ scores, general health $\left(r=0.206^{*}, p=0.027\right)$, lung health $\left(r=0.280^{* *}, p=0.003\right)$, medication $(r=0.142$, $p=0.130)$, skin health $\left(r=0.242^{*}, p=0.020\right)$, eye health $(r=0.176, p=0.089)$, in case of patients with sarcoidosis. We observed no significant associations between respiratory muscle MIP and general health $(r=0.004, p=0.961)$, lung health $(r=-0.019, p=0.836)$, and MEP and general health $(r=-0.059, p=0.525)$, lung health $(r=-0.030, p=0.745)$, in patients with sarcoidosis.

\section{Discussion}

In our study we found significant differences between the exercise capacity of patients with sarcoidosis and healthy controls. Moreover, significant differences regarding exercise capacity were observed between all the four stages of sarcoidosis. We also observed that both maximal inspiratory and expiratory pressure were lower in patients with sarcoidosis compared to healthy controls.

Health related quality of life can be significantly influenced in patients with sarcoidosis due to reduced physical capacity, dyspnea, lack of energy and anxiety and depression. ${ }^{18}$ Multifactorial influences such as systemic 


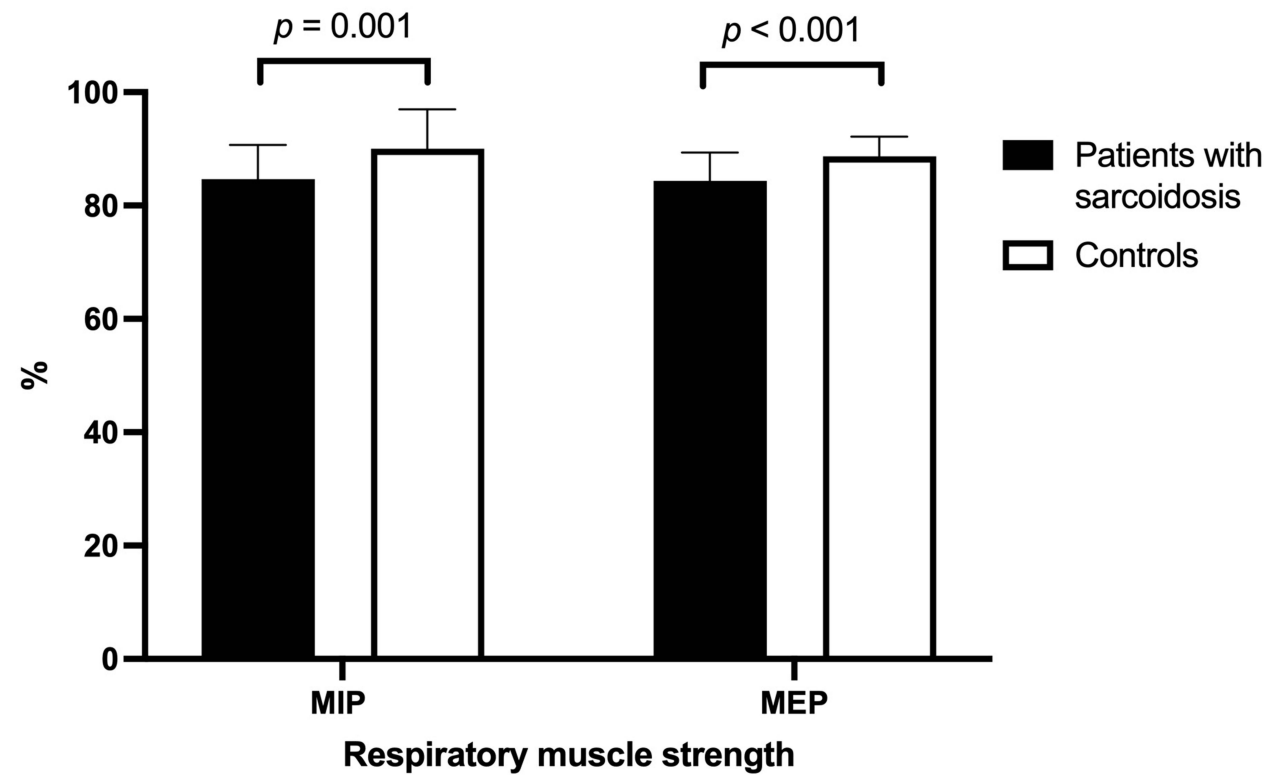

Figure I Respiratory muscle strength in patients with sarcoidosis and healthy controls.

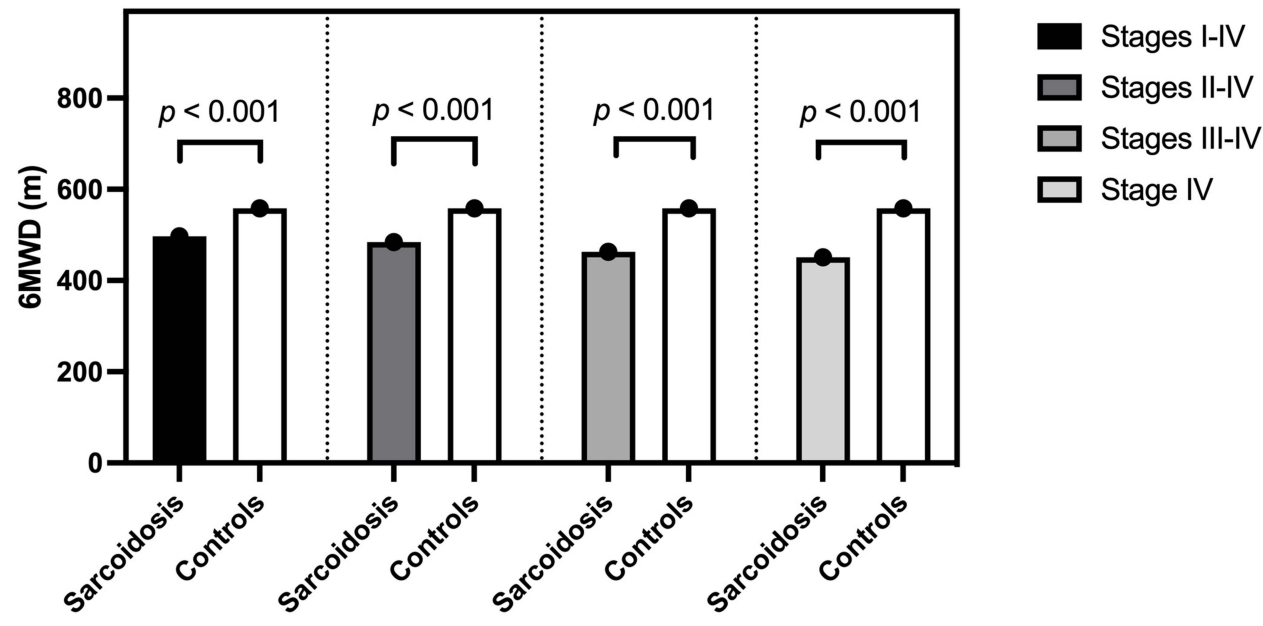

Figure 2 Exercise capacity in patients with sarcoidosis and controls. Patients with sarcoidosis were grouped by stage.

inflammation decreased pulmonary function, and others can lead to physical inactivity and loss of muscle conditioning. ${ }^{19}$

Table 3 Health Status of Patients with Sarcoidosis

\begin{tabular}{|l|c|}
\hline KSQ Scores & Patients $(\mathbf{n}=\mathbf{5 7})$ \\
\hline General Health & $83.00(76.00-88.00)$ \\
Lung Health & $81.00(73.00-86.00)$ \\
Medication & $88.00(81.00-92.00)$ \\
Skin Health & $100.00(96.00-100.00)$ \\
Eye Health & $100.00(97.00-100.00)$ \\
\hline
\end{tabular}

Notes: Data are presented as median (interquartile range). Abbreviation: KSQ, King's Sarcoidosis Questionnaire.
Marcellis et al demonstrated that exercise tolerance and muscle weakness are frequently reported, with substantial reduction of maximal inspiratory pressure (MIP).$^{20}$ The author noted that patients with sarcoidosis covered a shorter distance on the six-minute walk test, patients having reduced physical capacity by $20 \%$ compared to healthy subjects. These values were observed in over $45 \%$ of the sarcoidosis subjects. Moreover, he identified a positive association between inspiratory muscle strength and physical capacity. Inspiratory muscle strength (MIP) was reduced in $43 \%$ of the subjects. These findings were before observed by other authors who found reduced respiratory muscle strength and endurance time in 

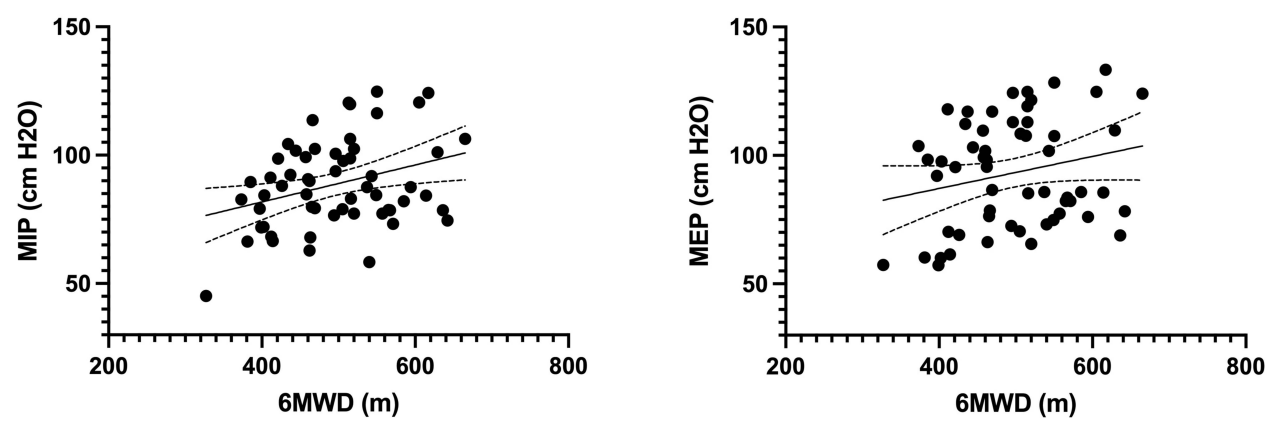

Figure 3 Association between exercise capacity and respiratory muscle strength in patients with sarcoidosis.

a small population of sarcoidosis patients with normal lung function. ${ }^{6}$ Compared to the study of Wirnsberger et al, ${ }^{6}$ we observed that maximal inspiratory and expiratory pressure were reduced in all our sarcoidosis patients. Moreover, we found significant correlations between respiratory muscle strength and physical capacity. An explanation for these findings could be that patients with reduced respiratory muscle strength require a greater use of the available muscles to overcome an external workload during breathing. Thus, these patients present exertional dyspnea leading to a reduced distance of $6 \mathrm{MWD}{ }^{4,6}$

In a much smaller study that included 15 patients with sarcoidosis and 14 healthy controls, authors have observed significantly lower daily step count compared to healthy subjects. Moreover, the step count was associated with the 6MWT distance. The 6MWD was lower in participants with sarcoidosis (mean (SD) $375 \mathrm{~m}$ ) compared to healthy controls (mean (SD) 487). The authors observed that the health status of the patients with sarcoidosis measured with the KSQ was moderate to severely impaired but they did not find significant correlations between the KSQ scores and 6MWD. ${ }^{21}$ Compared to this study, we observed that health status was moderate to severely impaired compared to the control group and we found significant associations between exercise capacity and KSQ scores.

A larger study that included 142 patients with sarcoidosis found that more than half of the patients walked in the 6MWT less than $400 \mathrm{~m}$ and 32 (22\%) patients walked less than $300 \mathrm{~m}$. The authors observed significant correlations between pulmonary function such as FVC and 6MWD and mentioned that also other factors such as activity aspect of quality of life questionnaire are important. ${ }^{22}$ In accordance with this study, we found significant association between exercise capacity measured by 6MWD and lung function measures in patients with sarcoidosis.
Compared to these studies, we have noticed that the physical capacity of patients with sarcoidosis was lower compared to the control group and between the sarcoidosis groups. Interestingly is that the mean distance in the 6MWT for both groups was higher compared to the aforementioned studies and it is clear that there are large differences in the results.

Some authors have pointed out that these differences in physical capacity cannot be fully explained by clinical features. It is mentioned that factors that could explain these differences could be the ethnicity and the socioeconomic and cultural aspects of the population. ${ }^{20}$ Although Romania is the second least active country in Europe, our patients with sarcoidosis remain more active compared to other patients in different countries.

A strong point of this articles is the fact that we included different stages of sarcoidosis and analysed physical capacity and respiratory muscle strength between patients and healthy controls.

A limitation of this study is that being retrospective we could not analyse what caused the limiting physical capacity and respiratory muscle strength. There still remain big differences between studies regarding physical capacity in patients with sarcoidosis and further work is needed to address this problem.

\section{Conclusion}

We observed that patients with sarcoidosis showed reduced quality of life, exercise capacity and respiratory muscle strength both among themselves and compared to the control group.

\section{Data Sharing Statement}

The authors confirm that all data underlying the findings are fully available without restriction. Data can be obtained after submitting a request to the hospital. 


\section{Ethical Approval and Consent to Participate}

This research was respecting the Declaration of Helsinki ethical principles for research regarding the safety of human subjects. The study design and contract forms were approved by the Ethics Committee of the Hospital of Infectious Diseases and Pneumophtisiology "Dr. Victor Babes", Timisoara (nr.6241/24/08/2020). Written informed consent was obtained from all the patients prior to the beginning of the study.

\section{Funding}

No funding was received for this work.

\section{Disclosure}

The authors declare that they have no competing interests.

\section{References}

1. Millward K, Fiddler CA, Thillai M. Update on sarcoidosis guidelines. Curr Opin Pulm Med. 2021;27(5):484-489. doi:10.1097/ MCP.0000000000000807

2. Valeyre D, Prasse A, Nunes H, Uzunhan Y, Brillet P-Y, MüllerQuernheim J. Sarcoidosis. Lancet. 2014;383(9923):1155-1167. doi:10.1016/S0140-6736(13)60680-7

3. Spruit MA, Thomeer MJ, Gosselink R, et al. Skeletal muscle weakness in patients with sarcoidosis and its relationship with exercise intolerance and reduced health status. Thorax. 2005;60(1):32-38. doi:10.1136/thx.2004.022244

4. Kabitz H-J, Lang F, Walterspacher S, Sorichter S, Müller-Quernheim J, Windisch W. Impact of impaired inspiratory muscle strength on dyspnea and walking capacity in sarcoidosis. Chest. 2006;130 (5):1496-1502. doi:10.1378/chest.130.5.1496

5. Baydur A, Alsalek M, Louie SG, Sharma OP. Respiratory muscle strength, lung function, and dyspnea in patients with sarcoidosis. Chest. 2001;120(1):102-108. doi:10.1378/chest.120.1.102

6. Wirnsberger RM, Drent M, Hekelaar N, et al. Relationship between respiratory muscle function and quality of life in sarcoidosis. Eur Respir J. 1997;10(7):1450-1455. doi:10.1183/09031936.97.10071450

7. Alhamad EH. The six-minute walk test in patients with pulmonary sarcoidosis. Ann Thorac Med. 2009;Apr(2):60-64. doi:10.4103/18171737.49414
8. Baughman RP, Sparkman BK, Lower EE. Six-minute walk test and health status assessment in sarcoidosis. Chest. 2007;132(1):207-213. doi:10.1378/chest.06-2822

9. Korenromp IHE, Heijnen CJ, Vogels OJM, van den Bosch JMM, Grutters JC. Characterization of chronic fatigue in patients with sarcoidosis in clinical remission. Chest. 2011;140(2):441-447. doi:10.1378/chest.10-2629

10. Saligan LN. The relationship between physical activity, functional performance and fatigue in sarcoidosis. J Clin Nurs. 2014;23(1516):2376-2378. doi:10.1111/jocn. 12490

11. Crouser ED, Maier LA, Wilson KC, et al. Diagnosis and detection of sarcoidosis. An official American thoracic society clinical practice guideline. Am J Respir Crit Care Med. 2020;201(8):e26-e51. doi:10.1164/rccm.202002-0251ST

12. Graham BL, Brusasco V, Burgos F, et al. 2017 ERS/ATS standards for single-breath carbon monoxide uptake in the lung. Eur Respir J. 2017;49(1):1-31. doi:10.1183/13993003.00016-2016

13. American Thoracic Society. ATS statement: guidelines for the six-minute walk test. Am J Respir Crit Care Med. 2002;166:111-117. doi:10.1164/ajrccm.166.1.at1102

14. Enright PL, Sherrill DL. Reference equations for the six-minute walk in healthy adults. Am J Respir Crit Care Med. 1998;158(5 Pt 1):1384-1387. doi:10.1164/ajrccm.158.5.9710086

15. Kera T. Measurement of respiratory muscle strength. Rigakuryoho Kagaku. 2002;17(4):265-271. doi:10.1589/rika.17.265

16. Borg E, Borg G, Larsson K, Letzter M, Sundblad B-M. An index for breathlessness and leg fatigue. Scand J Med Sci Sports. 2010;20 (4):644-650. doi:10.1111/j.1600-0838.2009.00985.x

17. Patel AS, Siegert RJ, Creamer D, et al. The development and validation of the King's Sarcoidosis Questionnaire for the assessment of health status. Thorax. 2013;68(1):57-65. doi:10.1136/thoraxjnl-2012201962

18. Drent M, Strookappe B, Hoitsma E, De Vries J. Consequences of sarcoidosis. Clin Chest Med. 2015;36(4):727-737. doi:10.1016/j. ccm.2015.08.013

19. Panagiotou M, Polychronopoulos V, Strange C. Respiratory and lower limb muscle function in interstitial lung disease. Chron Respir Dis. 2016;13(2):162-172. doi:10.1177/1479972315626014

20. Marcellis RGJ, Lenssen AF, Elfferich MDP, et al. Exercise capacity, muscle strength and fatigue in sarcoidosis. Eur Respir J. 2011;38 (3):628-634. doi:10.1183/09031936.00117710

21. Cho PSP, Vasudevan S, Maddocks M, et al. Physical inactivity in pulmonary sarcoidosis. Lung. 2019;197(3):285-293. doi:10.1007/ s00408-019-00215-6

22. Baughman RP, Lower EE. Six-minute walk test in managing and monitoring sarcoidosis patients. Curr Opin Pulm Med. 2007;13 (5):439-444. doi:10.1097/MCP.0b013e328273bc2b
International Journal of General Medicine

\section{Publish your work in this journal}

The International Journal of General Medicine is an international, peer-reviewed open-access journal that focuses on general and internal medicine, pathogenesis, epidemiology, diagnosis, monitoring and treatment protocols. The journal is characterized by the rapid reporting of reviews, original research and clinical studies across all disease areas. The manuscript management system is completely online and includes a very quick and fair peer-review system, which is all easy to use. Visit http://www.dovepress.com/ testimonials.php to read real quotes from published authors. 